\title{
Imported Malaria over Fifteen Years in an Inner City Teaching Hospital of Washington DC
}

\author{
Sri Lakshmi Hyndavi Yeruva ${ }^{1, *}$, Archana Sinha1', Mariam Sarraf-Yazdy', Jhansi Gajjala² \\ ${ }^{1}$ Department of Internal Medicine, ${ }^{2}$ Department of Infectious Disease, Howard University Hospital, Washington DC, USA
}

\begin{abstract}
As endemic malaria is not commonly seen in the United States, most of the cases diagnosed and reported are associated with travel to and from the endemic places of malaria. As the number of imported cases of malaria has been increasing since 1973, it is important to look into these cases to study the morbidity and mortality associated with this disease in the United States. In this study, we would like to share our experience in diagnosing and treating these patients at our institution. We did a retrospective chart review of 37 cases with a documented history of imported malaria from 1998 to 2012 . Among them, 16 patients had complicated malaria during that study period, with a mean length of hospital stay of 3.5 days. Most common place of travel was Africa, and chemoprophylaxis was taken by only $11 \%$ of patients. Travel history plays a critical role in suspecting the diagnosis and in initiating prompt treatment.
\end{abstract}

Key words: Plasmodium falciparum, Plasmodium vivax, imported malaria, teaching hospital, Washington DC

\section{INTRODUCTION}

Although endemic malaria is not seen in the United States of America (USA), majority of infections are caused by travel to or from endemic places of malaria transmission. The World Tourism Organization estimates that there were 1 billion international travelers in 2010, with notable increase in travel to Africa and South Asia [1]. With the increase of travel and increase of immigrants, number of cases of malaria in USA has also been increasing [2]. The prevention messages and health communication strategies have become even more important for protecting the traveling community from communicable diseases like malaria. Centers for Disease Control and Prevention (CDC), USA in 2010 reported a total of 1,688 cases of imported malaria in USA which was a $14 \%$ increase when compared to 2009 [3]. Imported malaria is defined as malaria acquired outside of USA and its territories. The aim of our study is to determine epidemiological characteristics and disease course of imported malaria in our hospital over the last 15 years.

\footnotetext{
- Received 7 October 2015, revised 24 March 2016, accepted 2 May 2016.

*Corresponding author (dryeruva24@gmail.com)

(C) 2016, Korean Society for Parasitology and Tropical Medicine

This is an Open Access article distributed under the terms of the Creative Commons Attribution Non-Commercial License (http://creativecommons.org/licenses/by-nc/4.0) which permits unrestricted non-commercial use, distribution, and reproduction in any medium, provided the original work is properly cited.
}

\section{MATERIALS AND METHODS}

A retrospective chart review of 15 years (1998-2012) was performed to determine the number of malaria cases in adults, i.e., individuals greater than 18 years of age at Howard University Hospital, a semi-urban hospital of District of Columbia, USA. The cases were identified based on discharge diagnosis of malaria and those that had definite diagnosis of malaria were chosen. The cases were defined as definite if the results of a blood smear with Wright-Giemsa stain were positive for malarial parasites. The degree of parasitemia quantitatively accessed at the time of diagnosis could only be retrieved for patients diagnosed and treated after 2008.

The records of 37 patients with malaria during the study period were reviewed for demographic data, including the age, sex, place of origin, travel history, initial diagnosis, and prophylaxis, along with prior history of malaria and month and year of admission. The various presenting symptoms and complications were recorded. Laboratory data were also reviewed and included complete blood cell counts, blood chemistries, and blood smears. Incubation period was defined as interval between dates of arrival at USA to the time of onset of symptoms. The initial diagnosis (usually recorded as the admitting diagnosis on the discharge summary) was recorded. The duration of illness from time of onset of symptoms to presentation in the hospital and their length of stay in hospital 
were recorded. The antimalarial treatment and response of the patient to the therapy was noted as well. The data were analyzed, and means and percentages were calculated. The results are presented as the histogram, pie chart, and line diagram. The results were compared to $\mathrm{CDC}$ data on malaria.

Travelers were defined as persons from USA travelled to endemic area of malaria and returned back. Immigrants with recent travel were defined as persons from endemic areas of malaria living in USA and travelled to endemic areas. Immigrants were defined as persons who moved to USA from malaria endemic areas.

\section{RESULTS}

All 37 cases in our study were of imported malaria. The diagnosis was confirmed by Giemsa stained thick and thin films of patient's peripheral blood. Their mean age was 38 years ranging from 20 to 66 years (Fig. 1), of which there were 24 males (65\%). African American accounted for the majority

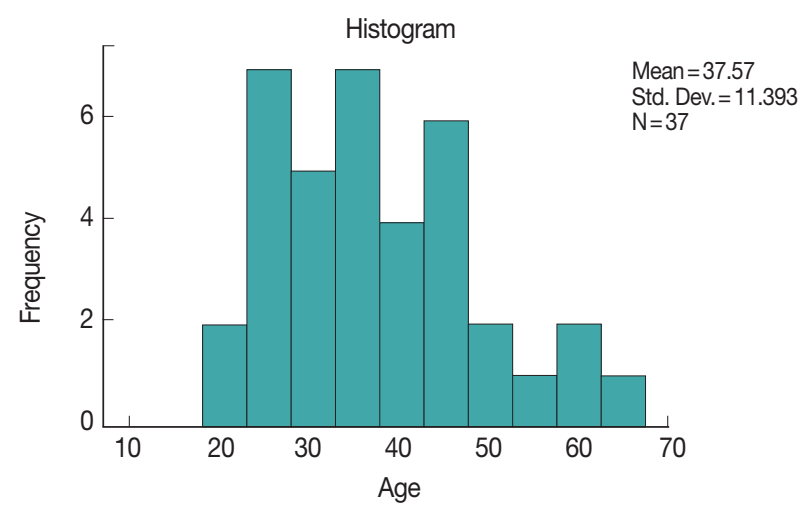

Fig. 1. Histogram showing the age distribution of malaria patients.

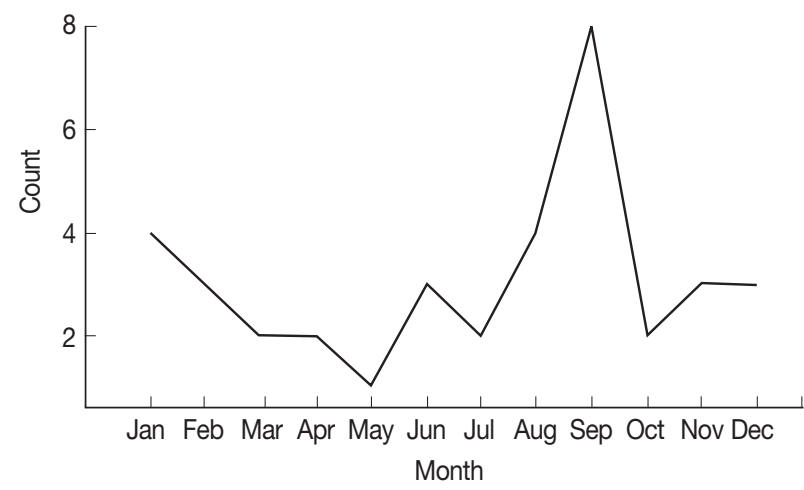

Fig. 2. Number of malaria patients by month from 1998 to 2012. with $26(70 \%)$, followed by Asian 1 (3\%), and race was unknown in 10 patients (27\%). The majority of cases, 8 (22\%), were seen in September (Fig. 2).

Eighteen (49\%) were travelers, and most common place of travel was Nigeria 20 (54\%) (Fig. 3). Only 4 (11\%) patients received chemoprophylaxis and a known past history of malaria was documented in $10(27 \%)$ of the cases. The majority (31/37) of the patients presented with fever and chills (84\%), followed by headache in 19 cases (51\%) and generalized body aches in 12 cases (32\%). The majority $(31 / 37 ; 84 \%)$ of cases were diagnosed with $P$. falciparum, and $3(8 \%)$ with $P$. vivax. The degree of parasitemia was available in 18 patients diagnosed and treated after 2008, which ranged from $0.001 \%$ to $0.3 \%$. All the 3 patients with P. vivax were treated with chloroquine with proguanil. Among the 31 patients with $P$. falciparum, $14(45 \%)$ were treated with quinine along with clindamycin/doxycycline, 13 (42\%) received treatment with mefloquine, and 4 (13\%) were given atovaquone-proguanil. The most common laboratory abnormality noted was thrombocytopenia in 27 (73\%) of patients, followed by abnormal liver function tests in $30 \%$. The majority (84\%) of patients had uncomplicated malaria, and complicated malaria was seen only in $16 \%$ of patients based on WHO criteria as shown in Table 1 . The mean length of hospital stay was 3.5 days with a mortality rate of $2.7 \%$.

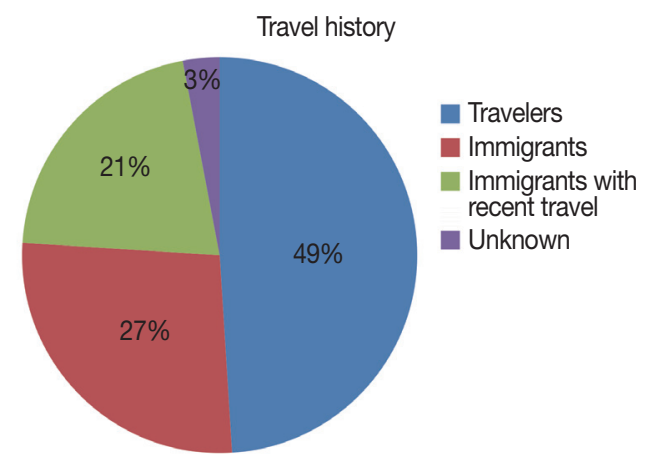

Fig. 3. Distribution of visiting places based on travel history.

Table 1. List of complications noted among our patients

\begin{tabular}{lc}
\hline Complication & Percentage \\
\hline Respiratory distress and acute respiratory distress syndrome & 10.8 \\
Renal Failure & 5.4 \\
Circulatory collapse & 5.4 \\
Coagulopathy and disseminated intravascular coagulopathy & 5.4 \\
Intravascular hemolysis/hemolytic anemia & 2.6 \\
Metabolic acidosis & 2.6
\end{tabular}




\section{DISCUSSION}

Malaria is a vector-borne, febrile illness caused by Plasmodium species which is transmitted by the bite of female anopheline mosquito. P. vivax, P. falciparum, P. ovale, P. malariae, and P. knowlesi are the 5 species of plasmodia, which have been identified to cause human malaria. Malaria due to P. falciparum is the most deadly, and it predominates in Africa. P. vivax is less dangerous but is more widespread, and the other 3 species are found much less frequently [4]. It is important to study malaria cases in USA as it is one of the preventable and treatable diseases, if current recommended interventions are properly implemented; prompt initiation of treatment for malaria is important to avoid mortality associated with it $[5,6]$.

Malaria can present similarly as any other febrile illness, and the most commonly presented symptom would be fever with chills which was seen in $84 \%$ of our patients. Other symptoms of malaria include nausea, vomiting, malaise, weakness, headache, diarrhea, abdominal pain, and coma [7]. The most common complication in our patients was thrombocytopenia compared to acute respiratory distress syndrome (ARDS), renal insufficiency, and cerebral malaria in a study done on imported malaria in US travelers [8].

The incubation period of malaria varies between 7 to 420 days based on the species. The incubation period for $P$. falciparum can vary from 9 to 14 days but also be as short as 7 days; the period for P. vivax is 12 to 17 days, and that for $P$. malariae is as long as 18 to 40 days $[9,10]$. Hypnozoites which are the dormant form can remain in the hepatocytes with $P$. vivax and $P$. ovale infections and can cause recurrent infection months or years after the initial infection. Our patients had a mean incubation period of 8 days as $94 \%$ of them had $P$. falciparum. We also found that $70 \%$ of imported malaria cases were travelers, and chemoprophylaxis was taken by only 4 (11\%) patients. One patient took mefloquine, 1 pyrimethamine, the other took doxycycline, and unknown in the last one. Similarly from the CDC data of imported malaria in USA, only $5 \%$ of the patients reported taking chemoprophylaxis. This fact confirms the importance of using more sufficient preventive measures for travelers specially travelers visiting friends and relatives in endemic areas [3]. Another interesting aspect of our study was that among the $28 \%$ of cases who were immigrants, a previous history of malaria was given in 10 patients (27\%), and all of them (100\%) had P. falciparum. So, it is important to make patients admitted for $P$. falciparum malaria aware of the importance of chemoprophylaxis on revisiting those endemic areas for malaria as 1 time infection does not provide any immunity towards recurrent malaria.

In our study, $94 \%$ and $8 \%$ of the cases were diagnosed with $P$. falciparum and $P$. vivax, respectively. In contrast to these findings, the distribution of imported malaria species in Aukland, New Zealand was reported to be 52.9\% P. falciparum and $47.1 \%$ P. vivax, where a major part of the investigated population was Indian migrants [11], and in another study by Stark et al. [12] reported 70\% of P. vivax as the main species of imported malaria cases from Pakistan to Germany [12]. These findings are consistent with the fact that $P$. falciparum predominates in African countries, and P. vivax predominates in Asian countries. Similar results were seen with imported malaria cases in USA that $72 \%$ from Africa were P. falciparum and $74 \%$ from Asia were P. vivax [13].

Traditionally, preparation of thick and thin peripheral blood smear with Giemsa stain and demonstration of malaria parasites in RBC is the gold standard of diagnosing malaria. As expertise is needed in diagnosing this infection from the smear especially in countries where the disease is not endemic, the diagnostic trial could result in false negative, and lack of resources can contribute to misdiagnosis in endemic countries [14]. Now a newer method, like rapid diagnostic test (RDT), is available to diagnose malaria by detecting circulating malarial antigen; however, it cannot aid in quantification or identification of Plasmodium species. So, still a blood smear is needed to confirm the diagnosis $[15,16]$. PCR is also available to diagnose malaria which is especially used in patients with a high suspicion for malaria with negative blood smears.

We also noted that the majority of cases were from West Africa with the peak month of incidence during September (Fig. 2), although CDC surveillance showed a peak incidence in July and January for patients who traveled to Africa due to the popular traveling time during holiday season in summer and early winter. However, this difference could be partially explained by travelers (including immigrants with recent travel) contributing to $70 \%$ of our cases, who usually return back at the end of summer vacation with reopening of schools. As per CDC data [3], it was found that the peak incidence of $P$. falciparum was seen in late August to early September as in our study where $94 \%$ of patients had $P$. falciparum. Similar to these findings, the end of summer was the peak time for malaria case reports in Germany among individuals returning or migrating from Pakistan [12]. 
Although this is a study involving a small number of patients, similar studies from a single institute were reported by Froude et al. [17] in which 51 imported malaria cases during 1986-1991 from their institute at Bronx, New York, USA were included, and Moore et al. [18] reported 59 imported malaria cases from their institute at Houston, Texas, USA during 19901993. In our study, with prompt diagnosis and initiation of treatment, a good prognosis was seen with a mean length of hospital stay of 3.5 days. Only 1 person (2.7\%) died of complicated malaria in whom diagnosis and treatment was delayed as travel history was not revealed by the patient at the time of admission. Malaria is not a common infection in USA, so travel history is a key for early diagnosis, and most importantly any patient who has been in an endemic area in the year preceding the onset of malarial symptoms should be evaluated for the disease $[2,7]$.

Due to an increase in international travels, imported malaria should be considered as one of the differentials in patients presenting with febrile illness from the endemic areas of malaria. As malaria is a treatable and preventable disease, there is increased need for creating awareness amongst travelers regarding chemoprophylaxis for effective prevention of this disease.

\section{CONFLICT OF INTEREST}

We have no conflict of interest related to this work.

\section{REFERENCES}

1. World Health Organization. World Malaria Report 2010. Geneva, Switzerland.

2. Jerrard DA, Broder JS, Hanna JR, Colletti JE, Grundmann KA, Geroff AJ, Mattu A. Malaria: a rising incidence in the United States. J Emerg Med 2002; 23: 23-33.

3. Mali S, Kachur SP, Arguin PM, Centers for Disease Control and Prevention (CDC). Malaria surveillance-United States, 2010. MMWR Surveill Summ 2012; 61: 1-17.

4. World Health Organization. World Malaria Report 2011. Geneva, Switzerland.
5. Greenberg A, Lobel HO. Mortality from Plasmodium falciparum malaria in travelers from the United States. Ann Intern Med 1990; 113: 326-327.

6. Walzer P, Gibson JJ, Schultz MG. Malaria fatalities in the United States. Am J Trop Med Hyg 1974; 23: 328-333.

7. Griffith KS, Lewis LS, Mali S, Parise ME. Treatment of malaria in the United States: a systematic review. JAMA 2007; 297: 22642277.

8. Agarwal A, McMorrow M, Arguin PM. The increase of imported malaria acquired in Haiti among US travelers in 2010. Am J Trop Med Hyg 2012; 86: 9-10.

9. Molineaux L. The epidemiology of human malaria as an explanation of its distribution, including some implications for its control. In Wernsdorfer WH, ed. Malaria, Principles and Practice of Malariology, Vol. 2. Edinburgh, Scotland. Churchill Livingstone. 1988, p 927.

10. Warrell DA. Clinical features of malaria. In Warrell DA, Gilles HM eds, Essential Malariology. 4th ed. New York, USA. Oxford University Press. 2002, p 192.

11. Camburn AE, Ingram RJ, Holland D, Read K, Taylor S. Imported malaria in Auckland, New Zealand. N Z Med J 2012; 125: 48-53.

12. Stark K, Schoneberg I. Increase in malaria cases imported from Pakistan to Germany in 2012. Euro Surveill 2012; 17: 20320.

13. Cullen K, Arguin Paul M, Centers for Disease Control and Prevention (CDC). Malaria surveillance-United States, 2012. MMWR Surveill Summ 2014; 63: 1-12.

14. Wangai LN, Karau MG, Njiruh PN, Sabah O, Kimani FT, Magoma G, Kiambo N. Sensitivity of microscopy compared to molecular diagnosis of $P$. falciparum: implications on malaria treatment in epidemic areas in Kenya. Afr J Infect Dis 2011; 5: 1-6.

15. Abba K, Kirkham AJ, Olliaro PL, Deeks JJ, Donegan S, Garner P, Takwoingi Y. Rapid diagnostic tests for diagnosing uncomplicated non-falciparum or Plasmodium vivax malaria in endemic countries. Cochrane Database Syst Rev 2014; 12: CD011431.

16. Beadle C, Long GW, Weiss WR, McElroy PD, Maret SM, Oloo AJ, Hoffman SL. Diagnosis of malaria by detection of Plasmodium falciparum HRP-2 antigen with a rapid dipstick antigen-capture assay. Lancet 1994; 343: 564-568.

17. Froude JR, Weiss LM, Tanowitz HB, Wittner M. Imported malaria in the Bronx: review of 51 cases recorded from 1986 to 1991. Clin Infect Dis 1992: 15: 774-780.

18. Moore TA, Tomayko JF Jr, Wierman AM, Rensime ER, White AC Jr. Imported malaria in the 1990s: a report of 59 cases from Houston, Texas. Arch Fam Med 1994; 3: 130-136. 